\title{
Sciendo
}

\author{
BULGARIAN ACADEMY OF SCIENCES
}

CYBERNETICS AND INFORMATION TECHNOLOGIES • Volume 19, No 2

Sofia • $2019 \quad$ Print ISSN: 1311-9702; Online ISSN: 1314-4081

DOI: $10.2478 /$ cait-2019-0014

\section{ISAR Imaging of a Rotating Asteroid Irradiated by Pulsar's Electromagnetic Emission}

\author{
Andon D. Lazarov \\ Burgas Free University, 8000 Burgas, Bulgaria \\ K. N. Toosi University, Tehran, Iran \\ E-mail:lazarov@bfu.bg
}

\begin{abstract}
The aim of the present study is imaging of moving objects, asteroids illuminated by continuous coherent wideband pulsar's signals. As pulsars are located on more than thousands of light years from Earth, objects crossing pulsars' emission beams are considered as second sources of electromagnetic waves, carrying object's shape and velocity information that can be extracted by application pulsar emissionbased inverse aperture synthesis. Inverse Synthetic Aperture Radar (ISAR) scenario, geometry and kinematics are analytically described. Models of pulsar signals and ISAR signals secondary emitted by asteroids are developed. White Gaussian noise of high level is added to the deterministic reemitted signal in order to approach the real signal scenario. Two-Dimensional (2D) Fourier transform for image extraction is applied. Special iterative noise removing procedure is suggested for asteroid's image enhancement. To verify mathematical model and imaging algorithms numerical experiments are carried out.
\end{abstract}

Keywords: Passive ISAR, ISAR imaging, ISAR signal model, additive Gauss noise depression algorithm.

\section{Introduction}

The pulsars born in consequence of massive stars' core collapse are neutron celestial bodies in the galaxy with powerful-pulsed electromagnetic emission. The pulsars are the densest form of matter in the Universe, exceeding the atomic nucleus density. The preserved angular momentum after the core's collapse causes rotation of the neutron star with period around 10 milliseconds and atomic precision. Rotating magnetic and electric fields accelerate clouds of charged particles causing emission of electromagnetic pulses in a wide spectrum of electromagnetic waves. Pulse profiles of 100 pulsars observed by the LOw-Frequency ARray (LOFAR) in two frequency ranges: high band (119-167 MHz, 100 profiles) and low band (15-62 MHz, 26 out of the 100 profiles) are presented in [1]. Pulsars' periodic electromagnetic emissions are highly stable over time. Emissions of pulsars with a millisecond period of rotation 
can be used for navigation purposes. It provides opportunity a spacecraft to autonomously determine its position with respect to an inertial origin. Properties of pulsars that make them attractive as potential natural navigation beacons and why a practical implementation looks most feasible in the X-ray band are discussed in [2]. An analysis of the instrumental sensitivity and signal processing in radio pulsar integrated navigation systems is provided in [3]. Based on stability of pulsars emission they can be used as highly accurate clocks, comparable in stability to atomic clocks. Millisecond X-ray pulsars, provide all the necessary ingredients for a passive galactic positioning system. This is known in astronautics as X-ray pulsar-based NAVigation (XNAV). An interplanetary navigation pulsar-based positioning system is presented in [5]. Pulsar signal processing algorithms for navigation purposes that consist of epoch-folding, matched filtering and detection are suggested in [6]. The algorithms are verified using real experimental data obtained from the radio observatory Dwingeloo, the Netherland. A hybrid detection algorithm based on energy and entropy analysis as an approach for spectrum sensing is considered in [7].

The signal power from pulsars is very low. In order to extract useful information a large amount of signal processing time is required. When the radio-waves of wideband pulsar emission pass through interstellar medium they get distorted and dispersed. In case of wide bandwidth emissions, this dispersion is quite substantial. It requires removing frequency dependency of the velocity of electromagnetic waves, i.e., to dedisperse the received signal before the informative signal and object itself have been detected.

The passive radar astronomy for observation, early warning and prevention of the asteroids' hit onto Earth surface is a completely new application area of pulsar signal emissions. However, the problem of asteroid detectability still remains, even more gets worst. Taking into account that the asteroid's velocity is around $30 \mathrm{~km} / \mathrm{s}$ the reliable distance to be detected and take counter measure is more than $500 \mathrm{~km}$, which poses serious issues in detection and processing of pulsar signals reemitted by asteroids. The detectability of the asteroid depends first on the echo's Signal-to-Noise Ratio (SNR), the ratio of echo power to the root-mean-square receiver noise. The SNR is a multifactorial parameter. It depends primarily on the asteroid's distance $R_{\mathrm{a}}$, diameter $D_{\mathrm{a}}$, and rotation period $P$; transmitter power $P_{\mathrm{tx}}$; the telescope's effective area $A$; the integration time $\Delta t$, i.e., $\mathrm{SNR} \sim\left(R_{\mathrm{a}}\right)^{-4}\left(D_{\mathrm{a}}\right)^{3 / 2} A^{2} P_{\mathrm{tx}} P^{1 / 2}(\Delta t)^{1 / 2}$ [8]. The integration time needed to achieve any given signal-to-noise ratio for a given target increases as $\left(R_{\mathrm{a}}\right)^{8} A^{-4}[8]$.

The pulsar is placed on a distance more than thousands light years from Earth and its pulsed radiation concentrated in a giant beam can be considered as an uninterrupted electromagnetic flow. In case a moving object, for instance, asteroid, crosses the area of an intensive electromagnetic field emitted by the pulsar, based on diffraction from the asteroid surface the illuminated asteroid can be regarded as a secondary source of electromagnetic emission.

The diffracted electromagnetic field is a superposition of electromagnetic waves reemitted from illuminated parts of the asteroid's surface. Measurements of time delay and Doppler frequency of the electromagnetic signals can be used for object's coordinates determination, velocity calculation and geometry extraction. Besides the 
intensity of the pulsar's signal is much lessed than the thermal noise intensity or other background signals pulsars' emissions can be still detected due to the periodicity of their beacons, providing that the integration time is long enough. Electromagnetic emissions with practical meaning having maximal intensity is received from pulsar Vela at a frequency around $400 \mathrm{MHz}$. The bandwidth of the pulsar's signal is 30-40 MHz. The atomic precision of pulsar signals period is based on the precision of the star's spinning period. For instance, European pulsar database issues the following data for pulsar B1937+21: period $1.558 \times 10^{-3} \mathrm{~s}$, pulse width $3.8 \times 10^{-2} \mathrm{~s}$, carrier frequency $1.4 \mathrm{GHz}$.

Physics of pulsars as sources of a huge electromagnetic radiation, properties of propagating electromagnetic waves through dispersed interstellar space, tools, methods and results of observations using pulsars' emissions performed by Jodrell Bank Observatory - the University of Manchester, and main steps of signal processing chain are discussed in [11-13]. In the present work, a conception to widen the application area of pulsar electromagnetic emissions is suggested using radar and synthetic aperture radar approach $[14,15]$. Though the power of emission is very low, around of mili-Jansky $\left(\mathrm{mJy}, 1 \mathrm{Jy}=10^{-26} \mathrm{~W} /\left(\mathrm{m}^{2} . \mathrm{Hz}\right)\right.$ ), in addition to autonomous spacecraft navigation the pulsar emission can be used, for shape extraction of objects crossing the pulsar's emission beam by applying inverse aperture synthesis principles.

Thus, based on reemitted electromagnetic waves the asteroid can not only be detected but also imaged. This is the reason the accent of the discussion to be on the geometry and kinematics description, signal modelling and asteroid imaging algorithms with application of passive inverse synthetic aperture radar principles $[16,17]$.

The remainder of the paper is organized as follows. In Section 2 geometry and kinematics of asteroid's passive ISAR imaging is described. In Section 3 a stepped frequency pulsar signal model, and in Section 4 a stepped frequency reemitted ISAR signal model are described. In Section 5 an algorithm of asteroid's ISAR image reconstruction is suggested. In Section 6 results of the numerical experiment are presented and discussed. In Section 7 conclusion remarks and recommendations for further research are given.

\section{Passive ISAR geometry and kinematics of asteroid imaging}

The scenario of the moving asteroid and imaged by a pulsar irradiation and Inverse Synthetic Aperture Radar (ISAR) principle is described in a coordinate system of observation $O x y z$ which origin coincides with the focus point of the radio telescope (Fig. 1). Due to the distance of the neutron stars, more than thousands light years away from Earth, and the assumption that the asteroid reradiates the pulsar emission, this geometry can be referred to passive inverse aperture synthesis. Based on the diffraction of the pulsar electromagnetic field on the asteroid surface the latter is regarded as a secondary emitter of an electromagnetic field carrying information with respect to velocity (Doppler frequency) and range (time delay) of the object, both determining the target image. 
Consider an asteroid as a flying rigid body with a rough surface rotating around its mass centre and moving against Earth. The object is depicted in its own coordinate system $O^{\prime} X Y Z$. Point $O^{\prime}$ is its mass centre. The position of an arbitrary point scatterer from the asteroid surface $P$ with discrete coordinates $i, j$, and $k$ on $X, Y$, and $Z$ axes, respectively, is defined with the position vector $\mathbf{R}_{i j k}=\left[X_{i j k}, Y_{i j k}, Z_{i j k}\right]^{\mathrm{T}}$, where [.] ${ }^{\mathrm{T}}$ stands for transposition.

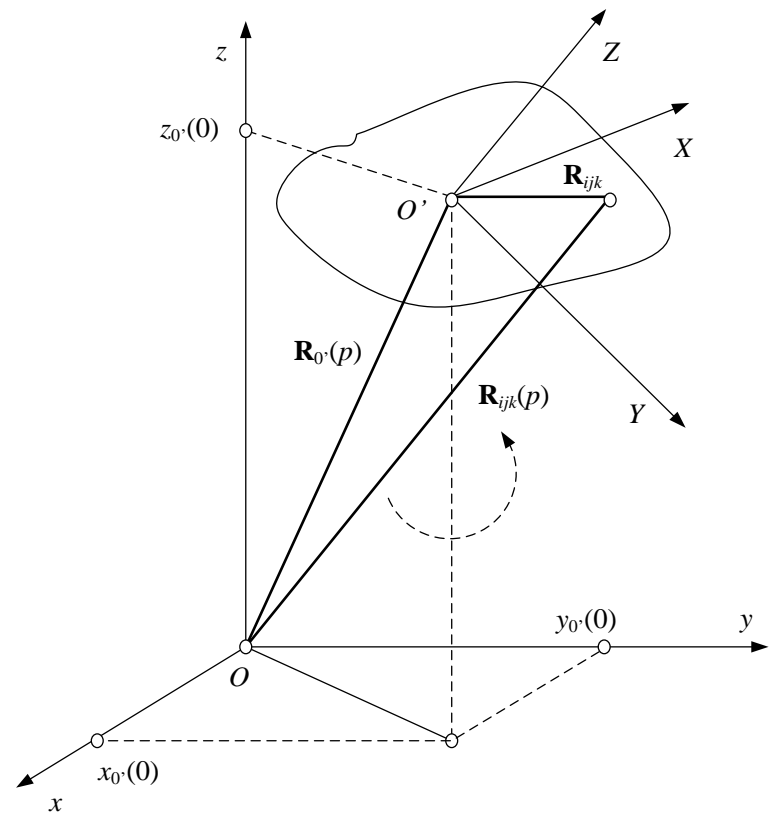

Fig. 1. Passive ISAR geometry

The mass centre's current position with coordinates $x_{0^{\prime}}(p), y_{O^{\prime}}(p)$, and $z_{O^{\prime}}(p)$ in coordinate system of observation $O x y z$ is defined by the time dependent distance vector $\mathbf{R}_{O^{\prime}}(p)=\left[x_{O^{\prime}}(p), y_{O^{\prime}}(p), z_{O^{\prime}}(p)\right]^{\mathrm{T}}$, where $p$ is the current measurements' moment. In case the asteroid is rotating around its mass centre and moving with a linear vector velocity $\mathbf{V}=\left[V_{x}, V_{y}, V_{z}\right]^{\mathrm{T}}$ the current position of the $i j k$ th point scatterer from the asteroid surface is defined with the distance vector $\mathbf{R}_{i j k}(p)=\left[x_{i j k}(p), y_{i j k}(p), z_{i j k}(p)\right]^{\mathrm{T}}$ expressed by equation

$$
\mathbf{R}_{i j k}(p)=\mathbf{R}_{O^{\prime}}(p)+\mathbf{A}(p) \mathbf{R}_{i j k},
$$

where $\mathbf{R}_{O^{\prime}}(p)=\mathbf{R}_{O^{\prime}}(0)+\mathbf{V} p T_{p}$ describes the rectilinear movement of the asteroid's mass centre, $\mathbf{R}_{O^{\prime}}(0)=\left[x_{O^{\prime}}(0), y_{O^{\prime}}(0), z_{O^{\prime}}(0)\right]^{\mathrm{T}}$ is the initial position vector of the mass centre, then $\mathbf{R}_{O^{\prime}}(p)$ in matrix form can be written as 


$$
\mathbf{R}_{O^{\prime}}(p)=\left[\begin{array}{c}
x_{O^{\prime}}(p) \\
y_{O^{\prime}}(p) \\
z_{O^{\prime}}(p)
\end{array}\right]=\left[\begin{array}{l}
x_{O^{\prime}}(0)+V_{x} p T_{p} \\
y_{O^{\prime}}(0)+V_{y} p T_{p} \\
z_{O^{\prime}}(0)+V_{z} p T_{p}
\end{array}\right],
$$

where: $T_{p}$ is the measurements time interval equal to burst repetition period; $V_{x}=V \cos \alpha, V_{y}=V \cos \beta, V_{z}=V \cos \delta$ are the coordinates of the linear vector velocity; $\alpha, \beta$, and $\delta$ are the vector velocity's guiding angles. In case the asteroid is moving rectilinearly on the mass-centre's line of sight, the velocity guiding angles are calculated by expressions as follows:

$$
\alpha=\arccos \left(\frac{x_{O^{\prime}}(0)}{\left|R_{O^{\prime}}(0)\right|}\right), \beta=\arccos \left(\frac{y_{O^{\prime}}(0)}{\left|R_{O^{\prime}}(0)\right|}\right), \gamma=\arccos \left(\frac{z_{O^{\prime}}(0)}{\left|R_{O^{\prime}}(0)\right|}\right) .
$$

The matrix operator $\mathbf{A}(p)$ is the rotation $3 \times 3$ Euler matrix with elements, functions of measurements moment $p$, i.e.,

$$
\mathbf{A}(p)=\left[\begin{array}{lll}
a_{11}(p) & a_{12}(p) & a_{13}(p) \\
a_{21}(p) & a_{22}(p) & a_{23}(p) \\
a_{31}(p) & a_{32}(p) & a_{33}(p)
\end{array}\right]
$$

where

$$
\begin{gathered}
a_{11}(p)=\cos \psi(p) \cos \varphi(p)-\sin \psi(p) \cos \theta(p) \sin \varphi(p), \\
a_{12}(p)=\cos \psi(p) \sin \varphi(p)+\sin \psi(p) \cos \theta(p) \cos \varphi(p), \\
a_{13}(p)=\sin \psi(p) \sin \theta(p), \\
a_{21}(p)=-\sin \psi(p) \cos \varphi(p)-\cos \psi(p) \cos \theta(p) \sin \varphi(p), \\
a_{22}=-\sin \psi(p) \sin \varphi(p)+\cos \psi(p) \cos \theta(p) \cos \varphi(p), \\
a_{23}=\cos \psi(p) \sin \theta(p), \\
a_{31}=\sin \theta(p) \sin \varphi(p), \\
a_{32}=-\sin \theta(p) \cos \varphi(p), \\
a_{33}=\cos \theta(p),
\end{gathered}
$$

and $\psi(p)=\psi+\omega_{z} p T_{p}$ is the time dependent yaw angle around $O z$ axis, $\varphi(p)=\varphi+\omega_{y} p T_{p}$ is the time dependent pitch angle around $O y$ axis, and $\theta(p)=\theta+\omega_{x} p T_{p}$ is the time dependent roll angle around $O x$ axis, $\omega_{x}, \omega_{y}$, and $\omega_{z}$ are the coordinates of the asteroid vector angular velocity, where $\psi, \varphi$, and $\theta$ are the initial angles of the asteroid orientation on yaw, pitch and roll directions.

In case the asteroid does rotate around $O z$ axis, i.e., $\psi(p)=\psi+\omega_{z} p T_{p}$ and does not rotate around $O x$ and $O y$ axes, i.e., $\omega_{x}=\omega_{y}=0$, and arbitrary oriented in the space, i.e., $\varphi(p)=\varphi=$ const,$\theta(p)=\theta=$ const the elements of the rotating matrix A can be rewritten as 


$$
\begin{gathered}
a_{11}=\cos \psi(p) \cos \varphi-\sin \psi(p) \cos \theta \sin \varphi, \\
a_{12}=\cos \psi(p) \sin \varphi+\sin \psi(p) \cos \theta \cos \varphi, \\
a_{13}=\sin \psi(p) \sin \theta, \\
a_{21}=-\sin \psi(p) \cos \varphi-\cos \psi(p) \cos \theta \sin \varphi, \\
a_{22}-\sin \psi(p) \sin \varphi+\cos \psi(p) \cos \theta \cos \varphi, \\
a_{32}=\cos \psi(p) \sin \theta, \\
a_{31}=\sin \theta(p) \sin \varphi, \\
a_{32}=-\sin \theta \cos \varphi, \\
a_{33}=\cos \theta .
\end{gathered}
$$

In case $\omega_{x}=\omega_{y}=0$, and $\varphi=\theta=0$ the asteroid rotates only around $O z$ axis, i.e., $\psi(p)=\psi+\omega_{z} p T_{p}$, then its rotating Euler matrix can be expressed as

$$
\mathbf{A}(p)=\left[\begin{array}{ccc}
\cos \psi(p) & \sin \psi(p) & 0 \\
-\sin \psi(p) & \cos \psi(p) & 0 \\
0 & 0 & 1
\end{array}\right] .
$$

The derived expressions from (1) to (6) are applied while modelling the geometry and kinematics of the asteroid's imaging scenario.

\section{Pulsar stepped frequency signal model}

Models of pulsars' signals can be created based on the structure of continuous electromagnetic fields emitted by pulsars. Based on radio telescope measurements of pulsar emissions $[6,9,10]$, the signals emitted by pulsars can be regarded as monochromatic if measurements are in signal frequency channel and/or polychromatic if measurements are in multiple frequency channels. The amplitudes of signals can be approximated by Gaussian and rectangular function, whereas the frequency structure as stepped frequency modulated with rectangular amplitude of a single frequency pulse in the burst, linear frequency modulated pulses with Gaussian envelop, etc.

The pulsar Crab, an uninterruptible emitter of very strong giant pulses in author's point of view is suited for asteroids tracking and passive ISAR imaging with ultra-high range resolution. The Crab's Main pulse consists of microbursts, each less than $1 \mu \mathrm{s}$ long, at $8-10 \mathrm{GHz}$ with bandwidth equal or greater than $2 \mathrm{GHz}[9,10]$. Based on these pulse parameters the Crab pulse emitted signal can be analytically described as a stepped frequency (SF) modulated or Linear Frequency Modulated (LFM) waveform.

In case the pulsar signal is modelled by the SF modulation waveform, a series of emitted pulse frequency bursts can be described by the expression

$$
s(t)=\sum_{p=0}^{N-1} \sum_{m=0}^{M-1} A_{m} \operatorname{rect} \frac{t-p T_{p}-m T}{T_{b}} \exp \left(j 2 \pi f_{m}\left(t-p T_{p}-m T\right)\right),
$$


where

$$
\operatorname{rect} \frac{t-p T_{p}-m T}{T_{b}}=\left\{\begin{array}{l}
1 \text { if } 0 \leq \operatorname{rect} \frac{t-p T_{p}-m T}{T_{b}}<1, \\
0 \text { otherwise, }
\end{array}\right.
$$

and $A_{m}$ is the amplitude of the pulses in the burst, $f_{m}=f_{0}+m \Delta f$ is the frequency of the pulse in the burst, $\Delta f$ is the frequency difference between adjacent frequency pulses in the burst, $f_{0}$ is the initial frequency in the burst, $T$ is the time width of the frequency pulse in the burst equal to the time repetition period of the frequency pulses in the burst; $m=0, \ldots, M-1$ denotes the pulse and/or frequency index, $M$ is the full number of pulses (frequencies) in each burst; $p=0, \ldots, N-1$ denotes the index of the emitted burst, $N$ is the full number of the frequency burst used for measurements.

\section{Stepped frequency ISAR signal modelling}

Based on the nature of Crabs pulsar emission the signal reradiated by the particular $i j k$-th point scatterer from the asteroid surface can be regarded as a sequence of time delayed SF signal bursts. In this sense, ISAR stepped frequency modulated continuous signal reradiated from the asteroid's surface can be described by equation

$$
\begin{aligned}
& s_{i j k}(t)=\sum_{p=0}^{N-1} \sum_{m=0}^{M-1} a_{i j k} \text { rect } \frac{t-p T_{p}-m T-t_{i j k}(p)}{T_{b}} \times \\
& \times \exp \left[j 2 \pi f_{m}\left(t-p T_{p}-m T-t_{i j k}(p)\right)\right],
\end{aligned}
$$

where $a_{i j k}$ is the reflectivity coefficient of the $i j k$-th point scatterer, $t_{i j k}(p)=\frac{\left|\mathbf{R}_{i j k}(p)\right|}{c}$ denotes the time delay from the $i j k$-th point scatterer, $c$ is the speed of the light. For the purpose of ISAR signal modelling inside the burst define the current time $t$ as $t=p T_{p}+m T+\hat{t}$, and $\hat{t}=t_{i j k \min }(p)+(m-r) T$, where $r=1+\left\lceil\frac{t_{i j k}(p)-t_{i j k \min }(p)}{T}\right\rceil$ denotes the number of the sample where signal from ijk-th point scatterer is detected, $\lceil x\rceil$ denotes the ceiling function that returns the smallest integer that is greater or equal to $x$. Then the expression (6) can be rewritten as

$$
s_{i j k}(t)=\sum_{p=0}^{N-1} \sum_{m=0}^{M-1} a_{i j k} \operatorname{rect} \frac{\hat{t}-t_{i j k}}{T_{b}} \exp \left[j 2 \pi f_{m-r}\left(\hat{t}-t_{i j k}(p)\right)\right] .
$$

The 2D ISAR signal reradiated from the whole asteroid surface in discrete form measured on cross-range $(p)$ and range $(m)$ coordinates can be described by equation

$$
S(p, m)=\sum_{p=0}^{N-1} \sum_{m=0}^{M-1} \sum_{i, j, k}^{I, J, K} a_{i j k} \operatorname{rect} \frac{\hat{t}-t_{i j k}}{T_{b}} \exp \left[j 2 \pi f_{m-r}\left(\hat{t}-t_{i j k}(p)\right)\right] .
$$


Complex additive Gaussian noise $n(p, m)$, is added to the deterministic signal using a standard mathematical procedure in order to model real ISAR data

$$
\hat{S}(p, m)=S(p, m)+n(p, m) \text {. }
$$

In accordance with a real ISAR scenario of the asteroid imaging the noise level exceeds the level of the deterministic signal. For the purpose of modelling and numerical experiments, the value of the signal to noise $(\mathrm{S} / \mathrm{N})$ ratio is accepted greater or equal to $-30 \mathrm{~dB}$.

\section{Asteroid's ISAR image reconstruction}

The phase structure of the received ISAR complex data consists of phases induced by the rectilinear radial and rotation movements. The velocity of the rectilinear radial movement is accepted constant and phases induced by it are considered as not informative. These phases can be removed by an appropriate radial motion compensation procedure, i.e., multiplication of $\hat{S}(p, m)$ with a phase term $\exp \left[-j 2 \pi f_{m} t_{O^{\prime}}(p)\right]$, i.e.,

$$
\tilde{S}(p, m)=\hat{S}(p, m) \exp \left[-j 2 \pi f_{m} t_{O^{\prime}}(p)\right],
$$

where $t_{O^{\prime}}(p)=\frac{\left|\mathbf{R}_{O^{\prime}}(p)\right|}{c}$ is the time delay of the ISAR signal reradiated by the asteroid mass centre.

After the aforementioned radial motion compensation, only phases induced by rotation movement remain and are regarded as most informative for asteroid imaging. Based on the plane wave front approximation of the electromagnetic wave propagation near the astronomical radio telescope, the complex image reconstruction can be decomposed into three main stages: 1) range compression of the base band (demodulated and radial motion compressed) ISAR signal; 2) cross-range (azimuth) compression of the range compressed ISAR complex signal; 3) final image extraction as a module and phase of the complex image:

1. Range compression of the base band ISAR signal by inverse Fourier transform

$$
\tilde{S}(p, \hat{m})=\frac{1}{M} \sum_{m=0}^{M-1} \tilde{S}(p, m) \exp \left(j \frac{2 \pi m \hat{m}}{M}\right),
$$

where $p=0, \ldots, N-1, \hat{m}=0, \ldots, M-1$.

2. Cross-range (azimuth) compression (complex image extraction) by inverse Fourier transform

$$
\tilde{I}(\hat{p}, \hat{m})=\frac{1}{M N} \sum_{p=0}^{N-1} \tilde{S}(p, \hat{m}) \exp \left(j \frac{2 \pi p \hat{p}}{N}\right),
$$

where $\hat{p}=0, \ldots, N-1, \quad \hat{m}=0, \ldots, M-1$ are the unknown cross-range and range coordinates of the point scatterers in the $2 \mathrm{D}$ image plane determined by correlation search procedures (13) and (14) maximizing the image function $\tilde{I}(\hat{p}, \hat{m})$. 
3. Final image extraction as a module $I(\hat{p}, \hat{m})$ and phase (argument) $\Phi[I(\hat{p}, \hat{m})]$ of the ISAR asteroid's complex image defined by the expressions

$$
\begin{gathered}
I(\hat{p}, \hat{m})=\left|\frac{1}{M N} \sum_{m=0}^{M-1} \tilde{S}(p, \hat{m}) \exp \left(j \frac{2 \pi p \hat{p}}{N}\right)\right| \\
\Phi[I(\hat{p}, \hat{m})]=\operatorname{Arg}\left[\frac{1}{M N} \sum_{m=0}^{M-1} \tilde{S}(p, \hat{m}) \exp \left(j \frac{2 \pi p \hat{p}}{N}\right)\right] .
\end{gathered}
$$

A complex image is extracted for further interferometric study of the asteroid's surface.

\section{Numerical experiment}

A numerical experiment has been carried out in order to illustrate a passive ISAR scenario of asteroid geometry, signal formation, complex kinematics and imaging. A model of continuous SF pulsar signal has been applied for the purposes of the experiment. ISAR signal parameters are carrier frequency $10^{10} \mathrm{~Hz}$ or wavelength 0.03 $\mathrm{m}$, burst frequency bandwidth $1.75 \times 10^{9} \mathrm{~Hz}$, burst time width $2 \times 10^{-6} \mathrm{~s}$, frequency step $1.36 \times 10^{7} \mathrm{~Hz}$, frequency pulse time width $1.56 \times 10^{-8} \mathrm{~s}$, burst repetition period $T_{p}=0.033 \mathrm{~s}$, number of range samples 128 , number of azimuth measurements 128 in one measurement segment, asteroid's initial coordinates of detection $x_{O^{\prime}}(0)=10 \mathrm{~km}, \quad y_{O^{\prime}}(0)=220 \mathrm{~km}, \quad z_{O^{\prime}}(0)=10^{3} \mathrm{~km}, \quad$ asteroid's module velocity $V=35.6 \mathrm{~km} / \mathrm{s}$, linear vector velocity angles $\alpha=1.06 \pi \beta=1.14 \pi, \gamma=1.85 \pi$, asteroid's angular position $\Psi(p)=\omega_{z} p \cdot T_{p}, \quad \theta=0, \quad \varphi=0$, angular velocity $\omega_{z}=0.1 \mathrm{rad} / \mathrm{s}$. Number of azimuth measurements $N_{p}=128$, dimensions of asteroid's grid resolution cell $\Delta X=\Delta Y=\Delta Z=1 \mathrm{~m}$. Number of range samples $N_{k}=128$. Taking into account that the ISAR signal from the asteroid is extremely weak in comparison with the additive Gaussian noise, the numerical experiment is carried out assuming signal to noise ratio is $(-30) \mathrm{dB}$. The following Matlab pseudocode is used to noise adding to the ISAR complex signal:

$$
\operatorname{Spm}(p, m)=\operatorname{awgn}(\operatorname{Spma}(p, m),-30, \text { "measured"); }
$$

An original solution to reduce the level of additive Gaussian noise in the complex image is suggested in the present work. It consists in consecutive additive summation of the obtained complex image (I) in accordance with the following Matlab pseudocode

$$
\begin{aligned}
& \mathrm{I}=0 \text {; } \\
& \text { for } r=1: 240 \\
& \text { Ia }=\text { ifft2(Stildapm); } \\
& \text { I =bsxfun(@plus,I,Ia); } \\
& \mathrm{A}=\operatorname{abs}(\mathrm{I}) \text {; } \\
& \mathrm{P}=\text { angle }(\mathrm{I}) \text {; }
\end{aligned}
$$


For the purpose of the experiment the number of complex images in the sum (number of iterations) mitigating the level of the additive white Gaussian noise is 240. Real and imaginary part of the pulsar ISAR signal reradiated by the asteroid and registered on range $(m)$ and azimuth $(p)$ coordinates are presented in Fig. 2. The real and imaginary part of the range compressed pulsar signal reradiated by the asteroid is presented in Fig. 3.

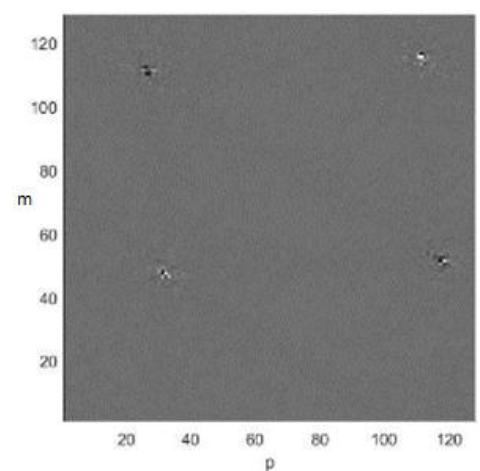

(a)

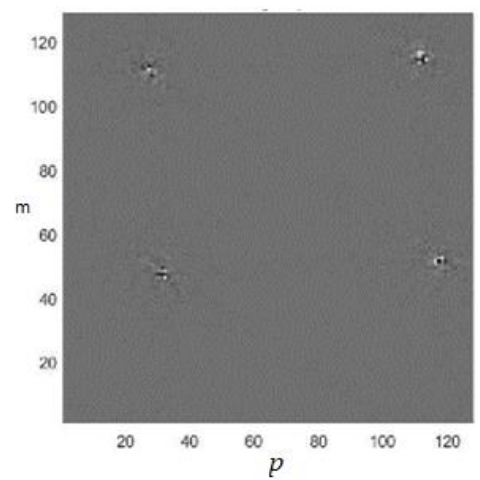

(b)

Fig. 2. ISAR signal $\tilde{S}(p, m):(a)$ real part; $(b)$ imaginary part

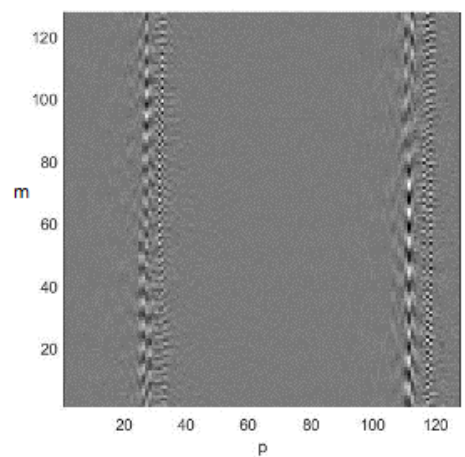

(a)

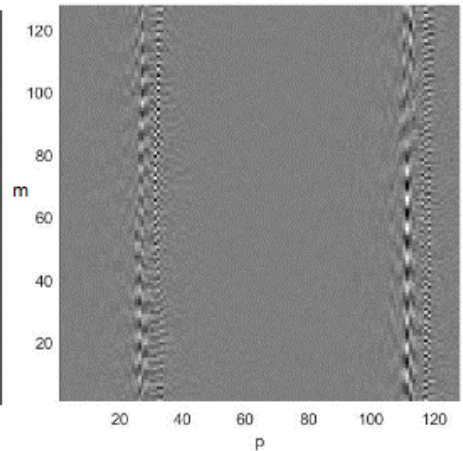

(b)

Fig. 3. Range compressed complex ISAR signal $\tilde{S}(p, \hat{m}):(a)$ real part; $(b)$ imaginary part

The final ISAR image of the asteroid after the first iteration $(r=1)$ is presented in Fig. 4. As can be seen there is no image in the frame. The evolution of the complex image quality due to additive noise removing procedure can be tracked out in Figs 5a-9a. The shape of the asteroid image appears after the iteration $r=34$ (Fig. 5a), and satisfactory image can be recognized in Fig. $9(r=240)$. 


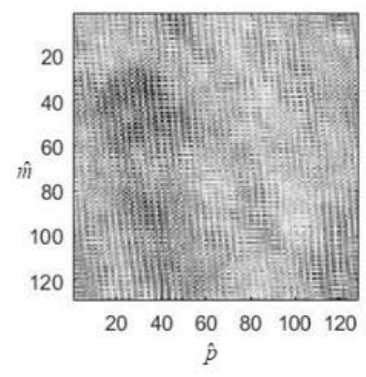

(a)

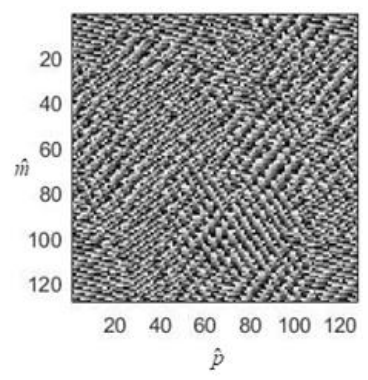

(b)

Fig. 4. Complex ISAR image $\tilde{I}(\hat{p}, \hat{m}), r=1:(a)$ module $I(\hat{p}, \hat{m}) ;(b)$ phase $\Phi[I(\hat{p}, \hat{m})]$

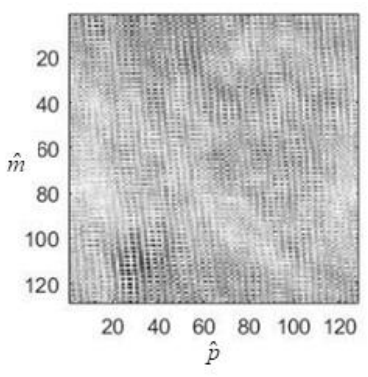

(a)

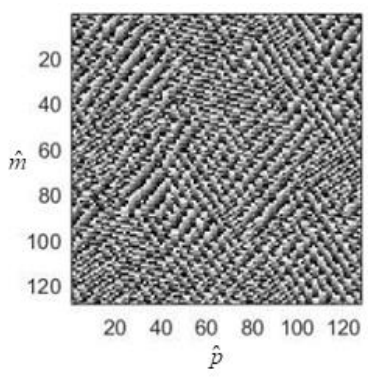

(b)

Fig. 5. Complex ISAR image $\tilde{I}(\hat{p}, \hat{m}), r=34:(a)$ module $I(\hat{p}, \hat{m}) ;(b)$ phase $\Phi[I(\hat{p}, \hat{m})]$

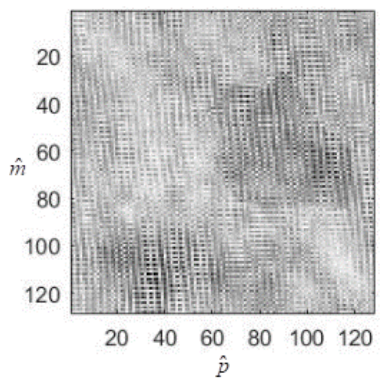

(a)

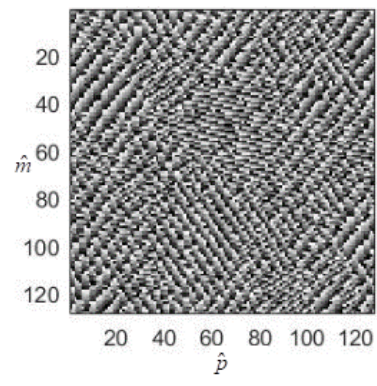

(b)

Fig. 6. Complex ISAR image $\tilde{I}(\hat{p}, \hat{m}), r=96:(a)$ module $I(\hat{p}, \hat{m}) ;(b)$ phase $\Phi[I(\hat{p}, \hat{m})]$

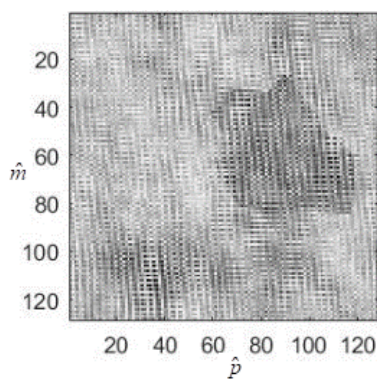

(a)

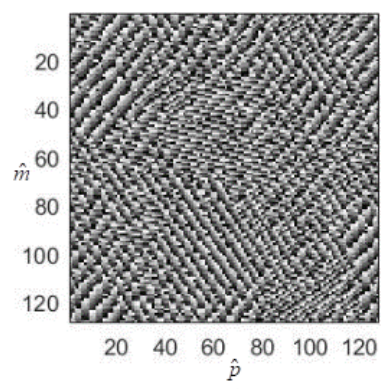

$(b)$

Fig. 7. Complex ISAR image $\tilde{I}(\hat{p}, \hat{m}), r=126:(a)$ module $I(\hat{p}, \hat{m}) ;(b)$ phase $\Phi[I(\hat{p}, \hat{m})]$ 
The evolution of the phases of the complex image based on noise removing can be tracked in Figs 5b-9b. The phases of the complex image can be used for further image processing of the asteroid as three-dimensional image reconstruction applying interferometric approach.

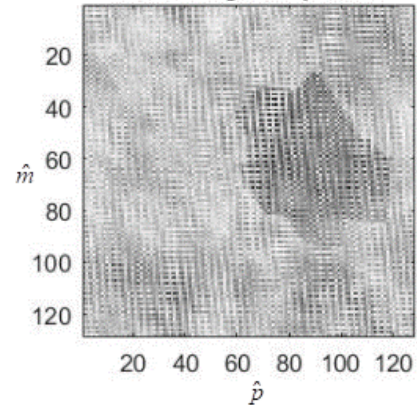

(a)

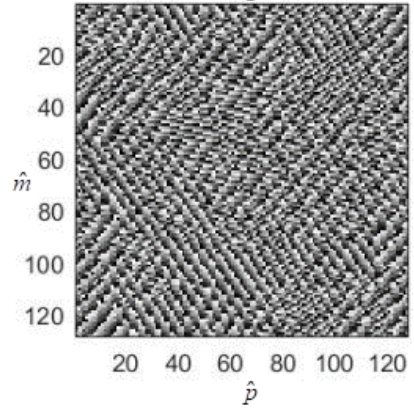

(b)

Fig. 8. Complex ISAR image $\tilde{I}(\hat{p}, \hat{m}), r=208:(a)$ module $I(\hat{p}, \hat{m}) ;(b)$ phase $\Phi[I(\hat{p}, \hat{m})]$

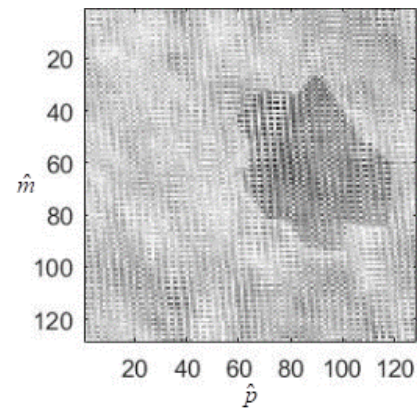

(a)

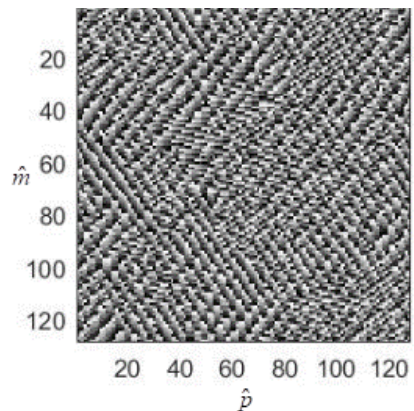

(b)

Fig. 9. Complex ISAR image $\tilde{I}(\hat{p}, \hat{m}), r=240:(a)$ module $I(\hat{p}, \hat{m}) ;(b)$ phase $\Phi[I(\hat{p}, \hat{m})]$

\section{Conclusion}

In the present work a new concept for asteroid imaging based on wide frequency band pulsar emission and ISAR principle has been developed. The geometry and kinematics of passive ISAR scenario with an asteroid moving rectilinearly and rotating around its mass centre has been analytically described. Geometry, kinematics and signal parameters during modelling processes are close to the real scenario of asteroids' observation and imaging. A conception for removing of the noise from the final asteroid image has been suggested and implemented in an image improving algorithm. It is to be pointed out that to implement the asteroid imaging algorithm with real pulsar data particular coherent pulsar emissions measurements have to be conducted without a presence of the asteroid in the emission field and with asteroid's presence in the emission field for the whole bandwidth of interest. First measurements have to be used as reference data in order to evaluate complex phase structure of the reradiated field from the asteroid. 


\section{References}

1. Pil i a, M., J. W. H e s s e 1. Wide-Band, Low-Frequency Pulse Profiles of 100 Radio Pulsars with LOFAR (The LOw-Frequency ARray). - Astronomy \& Astrophysics, Manuscript No 25196_ap c, ESO 2015, 2 November 2015, arXiv:1509.06396v2 [astro-ph.HE], 30 October 2015.

2. R a y, P. S., K. S. W o o d, B. F. Phli ps. Spacecraft Navigation Using X-ray Pulsars. Hulburt Center for Space Research, Featured Research 2006 NRL Review, pp. 95-102.

3. D o n g, J. Pulsar Navigation in the Solar System. - arXiv 0812.2635v3 [astro-ph], 4 February 2011.

4. Pulsar Positioning System: A Quest for Evidence of Extra-Terrestrial Engineering. - International Journal of Astrobiology, Vol. 2.0, Clément Vidal Center Leo Apostel Vrije Universiteit, Brussel Krijgskundestraat 33, 1160 Brussels, Belgium, 15 October 2017.

http://www.clemvidal.com

5. An Interplanetary GPS Using Pulsar Signals. MIT Technology Review. 23 May 2013. https://www.technologyreview.com/s/515321/an-interplanetary-gps-using-pulsarsignals/

6. K a b a k ch i e v, C., et al. Detection and Estimation of Pulsar Signals for Navigation. IRS 2015, 24-26 June 2015. DOI: 10.1109/IRS.2015.7226320.

7. Nik on ow i c z, J., P. Kubczak, Ł. M a t u s z e w s ki. Hybrid Detection Based on Energy and Entropy Analysis as a Novel Approach for Spectrum Sensing. - In: 2016 International Conference on Signals and Electronic Systems (ICSES'16), 5-7 September 2016, DOI: 10.1109/ICSES.2016.7593852.

8. O s t r o, S. J., et al. Asteroids III, Ch. Asteroid Radar Astronomy, 2002, pp. 151-168. https://echo.jpl.nasa.gov/asteroids/ast3_ostro+.pdf

9. E i l e k, J. A., T. H. H a n k in s. Radio Emission Physics in the Crab Pulsar, arXiv: 1604.02472v1 [Astro-ph.HE], 8 April 2016, Accepted for Publication in J. Plasma Phys.

10. H a n k i n, J. A. E i l e k, G. G o n e. The Crab Pulsar at Centimetre Wavelength, II. Single Pulses. - Astrophysics Journal, 10 December 2016, doi:10.3847/1538-4357/833/1/47.

11. Pulsars as Radio Sources. University of Manchester Jodrell Bank Observatory. http://www.jb.man.ac.uk/distance/frontiers/pulsars/section2.html

12. Pulsars as Tools. University of Manchester Jodrell Bank Observatory. http://www.jb.man.ac.uk/distance/frontiers/pulsars/section5.html

13. Searching for Pulsars, University of Manchester Jodrell Bank Observatory. http://www.jb.man.ac.uk/distance/frontiers/pulsars/section6.html

14. Douk ovska, L. Alternative Approaches for Target Velocity Estimation Using the Hough Transform in MIMO Radar Systems. - Cybernetics and Information Technologies, Vol. 11, 2011, No 1.

15. Ku dri a shov, V. V., A. Y. Garbar, K. A. Lukin, L. Mas likows ki, P. S a m c z yn ski, K. S. Kulpa. Fusion of Images Generated by Radiometric and Active Noise SAR. Cybernetics and Information Technologies, Special Issue on Information Fusion, Vol. 15, 2015, No 7.

16. S a m c z y n s ki, P., K. Kulpa. Passive SAR Imaging Using a Satellite Pulsed Radar as an Illuminator of Opportunity. - In: Proc. of 13th International Radar Symposium (IRS'12), 2012, pp.157-161.

17. O 1 i v a d e s e, D., E. G i u s t i, M. M a r t o r e 1 a et al. Passive ISAR with DVB-T Signals. - IEEE Transactions on Geoscience and Remote Sensing, Vol. 51, 2013, No 8, pp. 4508-4517.

Received: 17.12.2018; Accepted: 23.04.2019 